\title{
The Use of Wireless Sensor Network for Increasing Airport Safety
}

\author{
Jakub Kraus \\ ATM Systems Laboratory, \\ Department of Air Transport, Faculty of Transportation \\ Sciences, Czech Technical University \\ Horská 3, Praha 2, 128 03, Czech Republic \\ e-mail: kraus@fd.cvut.cz
}

\author{
Vladimír Němec \\ Department of Air Transport, \\ Department of Air Transport, Faculty of Transportation \\ Sciences, Czech Technical University \\ Horská 3, Praha 2, 128 03, Czech Republic
}

\author{
Petr Fajčík \\ Department of Air Transport, \\ Department of Air Transport, Faculty of Transportation Sciences, Czech Technical University \\ Horská 3, Praha 2, 128 03, Czech Republic
}

\begin{abstract}
This article deals with the use of wireless sensor networks for increasing safety at airports, respectively for replacing the current monitoring system to ensure safety. The article describes sensor networks and their applications to the identified processes and consideration of financial and safety benefits.
\end{abstract}

Keywords - WSN, sensor network, safety, airport

\section{INTRODUCTION}

Operational safety is needed to be constantly monitored and preferably improved. For these activities it is necessary to use a system, or technology that provides the best price/performance ratio and will achieve reasonable parameters in each of these categories. As the effort to increase the safety is continuous and never-ending process, it is appropriate to have also continuously working tracking system. That is why the use of sensor networks seems to be one possible solution.

\section{WIRELESS SENSOR NETWORKS}

Wireless sensor networks (WSN) consist of a group of small devices that are deployed in a specific area and communicate with each other wirelessly in order to monitor or control. By monitoring is meant gathering data using sensors and sending the measured values to superior system for processing, analysis and evaluation. [1] The proposed network is focused on the collection of one-dimensional data that is sent to the monitoring station. Unlike conventional sensor networks, video surveillance networks have two-dimensional data, i.e. video or photos.

The superior part of the sensor network is base station, where are data presented. The output of the network can be display with graphical representation of sensors. In case of detection, sensor that recorded event is identified, or image data are displayed.

Currently, sensor networks are not widely spread, but in recent years are subjected to intensive research.

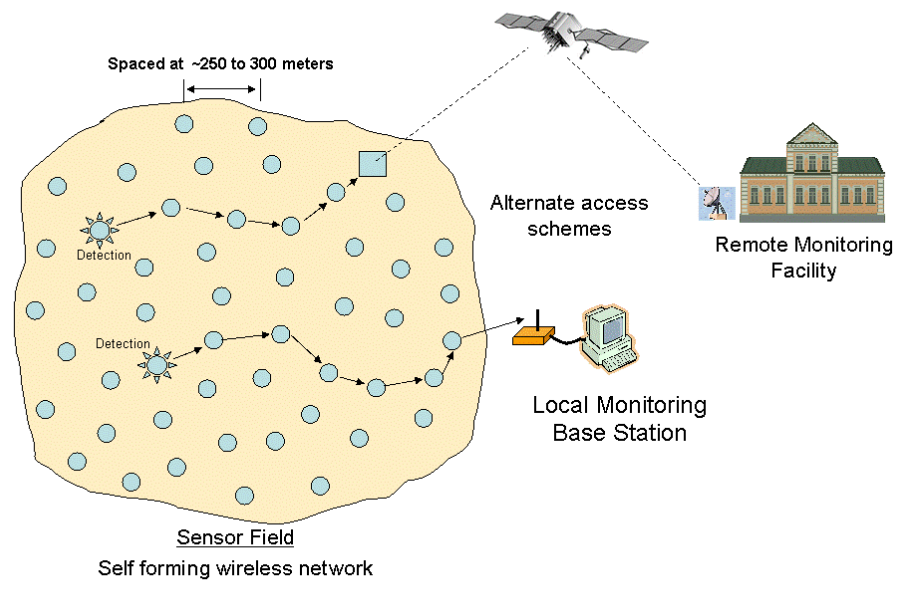

Figure 1. Wireless sensor network [3]

In general, all elements of the sensory networks should be simple, small and inexpensive devices with an emphasis on low power consumption. If the network requirements permit, network nodes should automatically switch to power saving mode after performing their task. A typical node is in standby mode for more than $99 \%$ of the time.

Devices used in wireless sensor network consist of the following components:

- Sensor - ICs sensing physical or chemical quantities and transform it into a digital signal

- Sensor board - a fundamental component to which are connected all the elements of a sensor network devices (sensors, power supply, antenna, radio module, ...)

- Power supply

- The antenna and radio module - to ensure "wireless" of wireless sensor networks 
The requirements for wireless sensor networks:

- Low power consumption

- Low cost

- Performance of sensors

\section{A. Standards for wireless sensor networks}

To achieve low economic demands and thus the widespread use of sensor networks, it is necessary to ensure standardization of network components. This allows the implementation of elements from different products and their mutual compatibility.

Success of wireless sensor network technology depends on the success of standardization efforts to unify the market, resulting in a large number of various mutually compatible devices. It will also manage to avoid the spread of ad hoc incompatible standards which, although may appear to be most appropriate for specific cases, overall reduce the possibility of developing wireless sensor market. The main subjects of interest are IEEE 802.15.4, at which basis is built ZigBee protocol that is being developed under the auspices of the ZigBee Alliance.

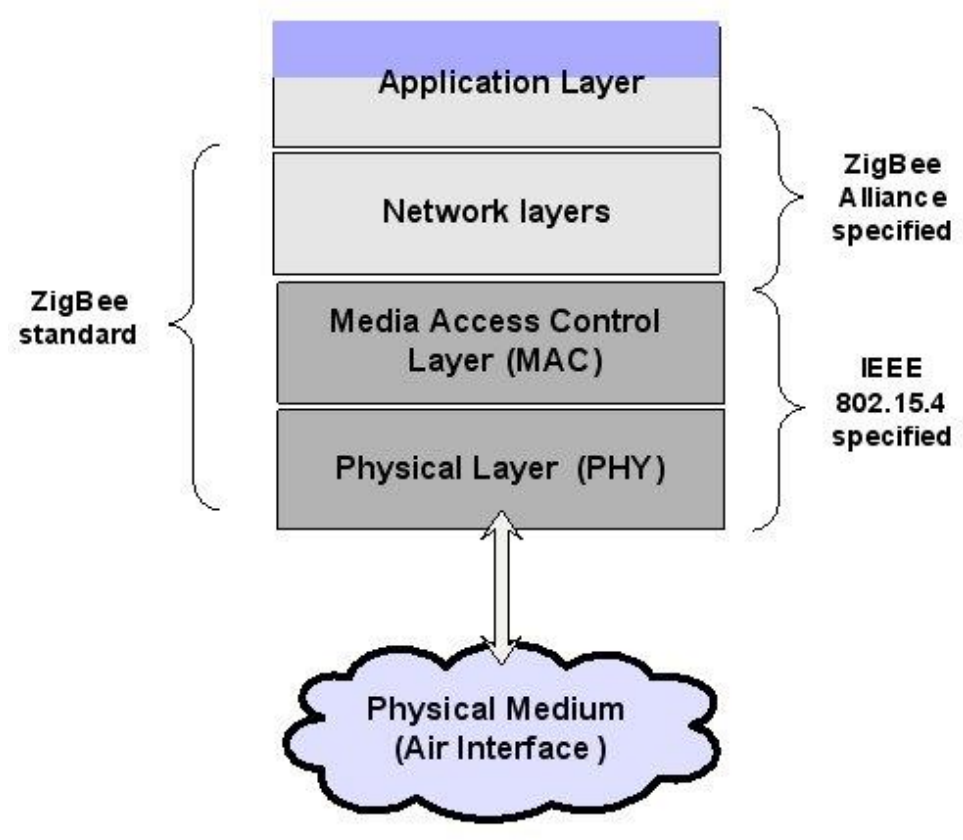

Figure 2. WSN Layers [2]

\section{B. Topology}

Although it is possible to choose from a variety of topologies - star, tree, peer2peer or mesh, from the perspective of airport safety is sufficient to use a simple star topology, because of the small airport dimensions (up to $7 \mathrm{~km}$ ).

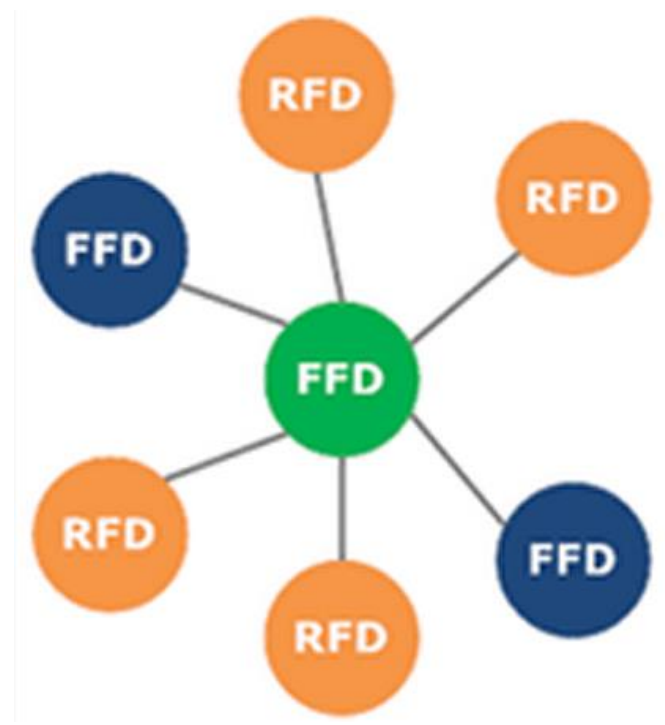

Figure 3. ZigBee star topology (FFD - fully functioning device - coordinator, FFD - fully functioning device, RFD - reduced function device)

\section{AIRPORT SAFETY PROCESSES}

All processes carried out at the airport should be safe, but not all are suitable to be monitored by using sensor networks. Identified processes where can be used sensor networks are in Table 1

TABLE 1. IDENTIFIED PROCESSES FOR USE OF WIRELESS SENSOR NETWORKS

\begin{tabular}{|l|}
\hline Runway incursion \\
\hline Runway overrun \\
\hline Providing protective area around the stand \\
\hline Fuelling \\
\hline Fire detection \\
\hline Towing aircraft \\
\hline Docking system for remote stand \\
\hline Collection of data for emergency situations \\
\hline
\end{tabular}

\section{APPLICATIONS OF WIRELESS SENSOR NETWORK FOR ENSURING SAFETY OF AIRPORT PROCESSES}

Sensor networks can only measure physical and chemical quantities. When using a visual sensor network is growing workload for signal processing techniques and thus vanishes financial advantage and simplicity.

\section{A. Runway Incursion}

Violation of runway area by an aircraft, vehicle, or person which is not allowed to enter at the time when air traffic is carried out is always very risky. Whereas ensuring the information about such violation is very simple thanks to the use of sensor networks and laser beams. Although laser beams cannot be considered as representative in the sensor networks, because they have not low power consumption, nothing can overcome their contribution in simplicity. 


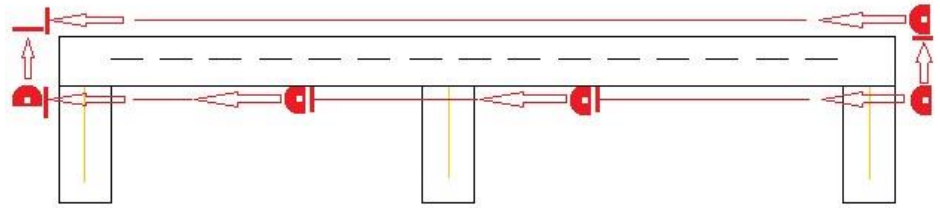

Figure 4. RWY Incursion ensured by sensor networks

In the design of this system is necessary to define what function it has to ensure, whether it is only for the information that "something is happening", or whether it should localize the intruder. This implies the required number of sensors. In Figure 4 is the principle with five sources and receivers, which is able to determine "the location" of the intruder.

For use at small aerodromess could be the first step in improving safety to have some information about the area violation and the position can be obtained simply by looking out the window. In this case, just one sensor and receiver need to be used together with three mirrors. (see Figure 5).

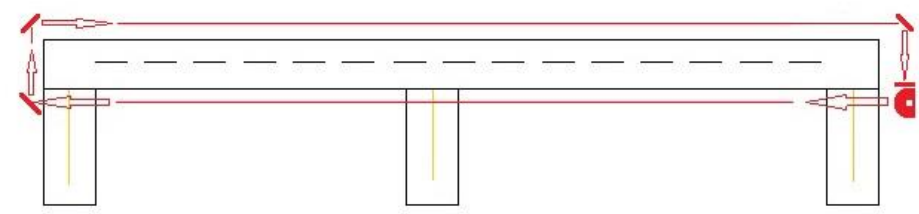

Figure 5. Low-cost RWY incursion

The same system can be used to provide information about runway overrun, or to provide the protection area around the stand.

\section{B. Fire detection}

Another obvious area for using sensor networks at airports is fire detection. This can be either in hangars or outdoors, with the fact that in the open space is necessary to have a sensor closer to the fire source or chemicals for overcome sensor threshold limit to get response.
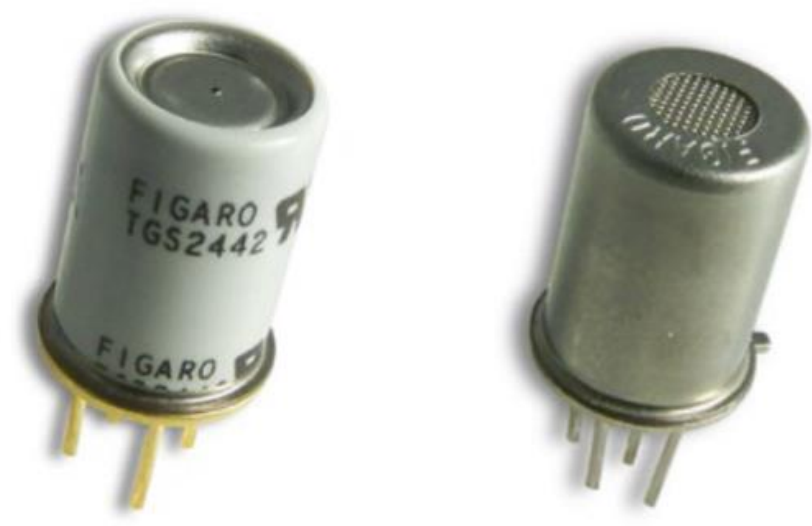

Figure 6. Sensors $\mathrm{CO}$ a $\mathrm{CO} 2$ [4]

The use of sensor networks to detect fire or only increased concentrations of $\mathrm{CO}, \mathrm{CO} 2$ and $\mathrm{NOx}$ can replace conventional fire detectors, but price comparison does not favour a sensor network. A better position would be to perform a complex safety/security system for building/hangar where it would be advantageous to ensure communication between multiple sensors.

Similar to fire detection may be the use of sensors for the detection of aviation fuel leakage. Sensors sensing the fumes from oil-based fuels could act as predictive fire detection system. This method could be used for refuelling aircraft to reduce the risk of fuel leakage.

\section{Towing aircraft and Docking system for remote stand}

Another way to make use of sensor networks for improve operating safety at the airport is to monitor activities involving the movement of aircraft, i.e. during towing and stopping at the remote stands without docking system.

Every year at every major airport happens several cases of towing where is improperly maneuvered with aircraft and the result is aircraft damage. These cases happen despite constantly improving regulations and staff training, and so the last option to improve is through technology.

During towing, blackbox could be attached to exposed parts, which would detect the entry of obstacles to the scope of his sensors (mostly spherical sector with radius $r$ ). The blackbox would include sensor board with obstacles sensor and when the obstacle will be recorded it would send a signal about the forthcoming danger to the central unit located on the tractor.

A similar system with opposite logic could function as docking system for remote stand. It would ensure the correct position of the aircraft on the surface, so the threat of damaging the aircraft from others will be avoided.

\section{V. „CBA“}

The current state of prices in sensor networks doesn't help to their implementation as the replacement of existing systems. However, their use as a technical improvement of processes will be beneficial, due to real cost savings from unrealized damages.

The cost of one "sensor place" consist of finance for sensor board, sensor, radio module and battery, thus generally 5000 CZK. However, costs for the repair of aircraft, plus the downtime, moves some zeros higher. From this perspective seems the use of sensor networks like a reasonable thing to do.

\section{CONCLUSION}

This article describes sensor networks and shows that they could not only be used for security purposes, but also for ensuring safety processes at airports. Although these are not too many, we identified eight where the contribution of sensor network can mean substantial cost savings.

Since sensor networks are very diverse, their usage can be almost anywhere, so it is certain that there are more processes where sensor networks can be used, therefore it will be useful to completely analyse airport to identify the places for sensor 
networks and subsequently create CBA for confirming that it is good idea.

\section{ACKNOWLEDGEMENTS}

This paper was supported by the Grant Agency of the Czech Technical University in Prague, grant No. SGS12/165/OHK2/2T/16.

\section{REFERENCES}

[1] Callaway, Edgar H, Jr: Wireless Sensor Networks: Architectures and Protocols.

[2] Collotta, M., G. Pau, V.M. Salerno and G. Scatá: Wireless Sensor Networks to Improve Road Monitoring DOI: 10.5772/ Available at $<$ http://www.eetimes.com/document.asp?doc_id=127411 5>

[3] Wireless sensor network (WSN) [online]. [cit. 2013-0808]. Available at $<$ http://embedsoftdev.com/embedded/wireless-sensornetwork-wsn/>

[4] Events board technical guide.pdf [online]. [cit. 2013-0808]. Available at $<$ http://www.libelium.com/development/waspmote/docu mentation/events-board-technical-guide/> 